\section{EPIDEMIC INFLUENZA}

\section{A CLINICAL POINT OF VIEW*}

BY

\section{H. STUART-HARRIS, M.D., M.R.C.P.}

(From the National Institute for Medical Research, Hampstead, London, N.W.3)

Although the filterable virus of epidemic influenza was first isolated in 1933, no work has been carried out until recently which has combined a clinical and a pathological study of the virus disease. During 1936 we investigated clinically and attempted to isolate virus from four localized epidemics which had been diagnosed as influenza. We did not recover virus, and we obtained serological evidence that the virus was not concerned. Then in December, 1936, the widespread influenza epidemic afforded us an opportunity of correlating the clinical events in man with the isolation of the virus from individual patients. Most of the clinical work in these combined investigations was for several reasons carried out upon patients in the Services. Chief among these were the facts that the men were all healthy before the illness, so that the picture of the disease was clean; and, secondly, that the patients were admitted to hospital early in the course of the disease, which enabled the whole illness to be studied and facilitated the recovery of the virus.

The principal question throughout the work has been whether influenza virus infection in man constitutes a clinical entity or not. Experience in a further epidemic and in the civil population will have to be gained before our tentative conclusion that it does constitute an entity can be proved. The evidence for believing that we were dealing with an entity during the recent epidemic was threefold. First, the disease presented a remarkably uniform clinical picture, case after case exhibiting similar features. It is true that the severity of the disease varied somewhat in the different areas, but the general picture was everywhere the same. Secondly, this characteristic clinical picture was correlated with the recovery of virus from the throat in a high proportion of cases. We were not able to test every patient owing to the number of ferrets required, but representative individuals from groups of similar clinical type were chosen and virus was isolated from twelve out of fifteen patients with straightforward influenza who were tested and whom I saw myself. Thirdly, the clinical picture we attempted to delineate during the 1936-7 epidemic differed from that seen in previous epidemics which we had studied and from which virus was not obtained.

\section{Variations in Clinical Type and Experimental Analogies}

During the influenza epidemic certain analogies became evident between influenza virus infection in man and the experimental disease in ferrets and mice-particularly between man and the ferret. Influenza virus can produce in the ferret either a short fever with lassitude, nasal symptoms, and nasal lesions, or an illness accompanied by the development of lung lesions varying in extent from small patches of bronchiolitis and atelectasis to fatal pneumonic consolidation. Similarly, although the more usual illness in man that was of virus aetiology was a three-day or four-day fever with constitutional symptoms and signs of upper respiratory tract infection, other cases from which virus was obtained had signs of chest disease.

* Read in opening a discussion in the Section of Pathology, Bacteriology, and Immunology at the Annual Meeting of the British Medical Association, Belfast, 1937.
The commonest type of chest abnormality was a basal condition with rales and suppressed breath sounds, which I believe to be due to a bronchiolitis with patchy atelectasis or scattered areas of consolidation. Virus was isolated from each of five patients tested who had signs of this condition. Frank pneumonic cases, however, did not usually yield virus from garglings and sputum by the time that lung signs were definite, but bacteria such as pneumococci were then recovered; however, one case of pneumonia yielded virus from garglings and sputum on the fifth day of the disease, when consolidation of lobar distribution was present, and virus was also recovered from the lungs in three rapidly fatal cases of pneumonia.

An interesting example of the similarity of the infective process in man and the ferret was seen in connexion with the pyrexia. Several different types of fever were observed in man, including a single peak, a remittent fever, and a fever with two peaks. This latter type, which was seen in 25 per cent. of patients, was of special interest, because it resembled the diphasic fever seen so commonly in ferrets infected with influenza virus.

In addition to the typical cases of influenza a number of mild infections and afebrile patients with symptoms were seen in some of the epidemic areas. Two afebrile patients were tested for virus, but this was not recovered, and although it is possible that such cases represented abortive infections by the virus, such as have occurred very occasionally in the ferret experimentally, we have no proof that the human infections were so caused. Suggestive serological evidence was obtained that subclinical infections by the virus can occur in man; and if this is so, then afebrile patients with symptoms would logically form the next grade of severity to such silent infections.

Finally, some patients presented the ordinary picture of the epidemic disease but suffered from repeated vomiting at the onset of illness. One such case was tested, and virus was recovered from the throat, but another patient who suffered from diarrhoea as well as vomiting and had no respiratory symptoms did not yield virus. No other cases with intestinal symptoms were seen throughout the epidemic, and so, although a form of infection with gastric symptoms at the onset can be due to the virus, there is no proof of the existence of a separate and distinct gastro-intestinal form of the virus infection.

\section{Differentiation of Epidemic Influenza and "Febrile Catarrhs"}

With regard to the question of differentiating the virus disease from other conditions resembling epidemic influenza, the form of the epidemic may help. At Chatham where cases from the naval barracks were studied, an epidemic occurred in November, 1936, of tonsillitis and of a pharyngo-laryngo-tracheitis disease. Influenza virus was not recovered at this time, but in January, 1937, a sharp epidemic occurred and virus was now found. The explosive onset of the influenza epidemic contrasted with the slow rise of the previous epidemic from the endemic respiratory infections. Again, the influenza epidemic was soon over in each area and rarely lasted longer than a fortnight; secondary waves were seen, particularly at Chatham, but in each case these were due to the spread of the epidemic among new entries to the barracks, either those returning from leave or visitors to the port. Finally the influenza epidemic consisted of cases of similar clinical type though varying in severity, while the previous Chatham epidemic included two groups of cases, one with frank tonsillitis and the other with pharyngo-laryngo- 
tracheitis. For these latter cases, which yielded no evidence of influenza virus infection, we propose to use the term "febrile catarrhs." .It must be emphasized that the name refers to a group of conditions forming the scrapheap of epidemic respiratory infections resembling influenza but of unknown aetiology, and not to a specific condition.

On clinical grounds the first symptoms of illness which are complained of show a sharp contrast between epidemic influenza and febrile catarrh. Table I

TABLE I.-Symptoms at Onset

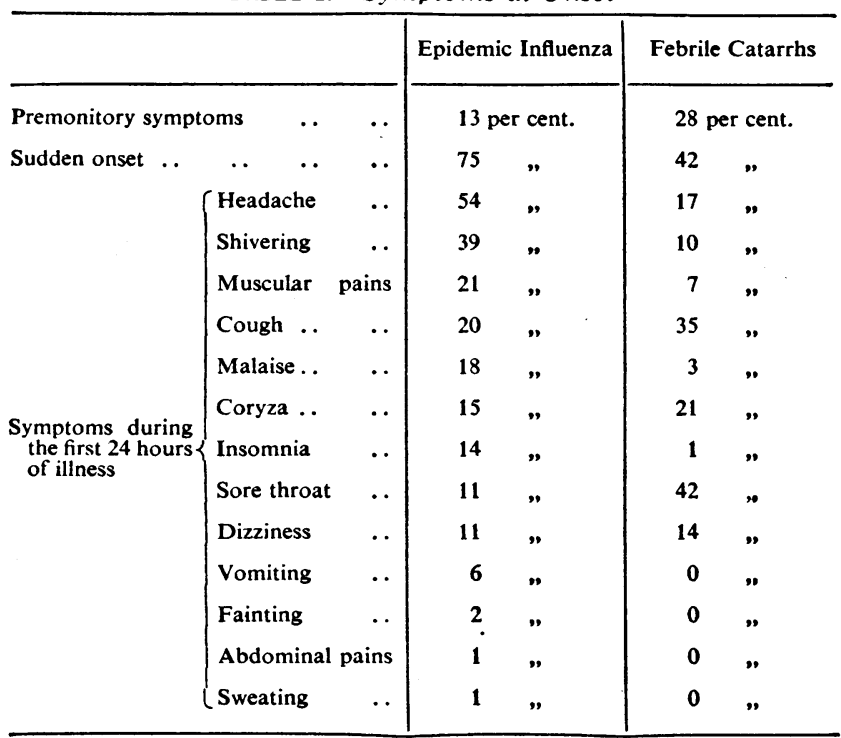

shows an analysis of the symptoms at the onset in eightyfour cases of straightforward influenza contrasted with those in twenty-eight cases of febrile catarrh.

TABLE II.-Symptoms During the Course of the Fever

\begin{tabular}{|c|c|c|c|c|c|c|c|c|}
\hline & & & & & Epidem & Influenza & Febril & Catarrhs \\
\hline & .. & . & . & .. & 91 & cent. & 82 & cent. \\
\hline Headache & . & $\cdots$ & $\cdots$ & . & 87 & $"$ & 64 & $"$ \\
\hline Anorexia & .. & $\cdots$ & $\cdots$ & .. & 77 & $"$ & 70 & " \\
\hline Shivering & .. & $\cdots$ & $\cdots$ & $\cdots$ & 74 & $\bullet$ & 70 & " \\
\hline Coryza or nas & al obst & ructi & & $\cdots$ & 73 & " & 70 & $"$ \\
\hline Cough .. & . & $\cdots$ & .. & .. & 71 & $"$ & 86 & $\bullet$ \\
\hline Dizziness & .. & $\cdots$ & $\cdots$ & .. & 62 & $"$ & 64 & " \\
\hline Muscular pair & & $\cdots$ & $\cdots$ & $\cdots$ & 51 & " & 53 & " \\
\hline Sore throat & . & $\cdots$ & $\cdots$ & .. & 43 & " & 80 & " \\
\hline Ocular symptc & oms & $\cdots$ & $\cdots$ & $\cdots$ & 33 & " & 21 & " \\
\hline Insomnia & . & $\cdots$ & $\cdots$ & .. & 32 & $"$ & 40 & $"$ \\
\hline Sweating & . & .. & .. & .. & 31 & $"$ & 50 & $"$ \\
\hline Expectoration & .. & $\cdots$ & $\cdots$ & $\cdots$ & 30 & " & 50 & " \\
\hline Pains in chest & .. & $\cdots$ & $\cdots$ & .. & 24 & " & 40 & " \\
\hline Epistaxis & . & $\cdots$ & .. & .. & 21 & " & 21 & " \\
\hline Constipation & .. & $\cdots$ & .. & .. & 21 & " & 28 & " \\
\hline Nausea .. & .. & $\cdots$ & .. & .. & 21 & " & 3 & $"$ \\
\hline Vomiting & $\cdots$ & $\cdots$ & .. & . & 11 & $"$ & 28 & " \\
\hline Dyspnoea & .. & $\cdots$ & .. & . & 11 & " & 0 & " \\
\hline Abdominal pa & & $\because$ & .. & . & 8 & $"$ & 28 & " \\
\hline Hoarseness of & voice & $\cdots$ & .. & .. & 6 & " & 40 & " \\
\hline Fainting & .. & $\cdots$ & .. & . & 4 & " & 0 & " \\
\hline Faintness & $\cdots$ & $\cdots$ & . & . & 2 & $"$ & 3 & " \\
\hline Diarrhoea & . & $\cdots$ & . & . & 0 & " & 3 & $"$ \\
\hline
\end{tabular}

Premonitory symptoms such as coryza, sore throat, or cough are uncommon in influenza, which starts abruptly in the midst of good health. In febrile catarrh, however, the disease begins frequently with a cold, and fever follows in some days' time. Moreover, the first symptoms in influenza are of the general or constitutional type and not of a respiratory nature. Considering the symptoms throughout the fever (Table II) the contrast is less striking. Again, however, general symptoms dominate the illness in influenza, and local respiratory ones predominate in febrile catarrh. Certain symptoms deserve special mention. Cough was short and dry in influenza, but paroxysmal, tearing, and barking in febrile catarrh. In association with the cough, substernal soreness of the chest was more striking in febrile catarrh, which is often a tracheitis. Sore throat is not a feature of influenza, but is constant in febrile catarrh. Laryngitis is rarely severe in influenza, but a very hoarse voice is common in febrile catarrh. Certain other symptoms, however, such as muscular pains and epistaxis, show no difference in frequency in the two diseases.

The differences are summarized in Table III, and there is no doubt that the two conditions show a contrast when the cases are considered in groups. The difficulty arises when individual patients are seen. Then the general

TABLE III.-Differential Diagnosis of Epidemic Influenza and Febrile Catarrhs

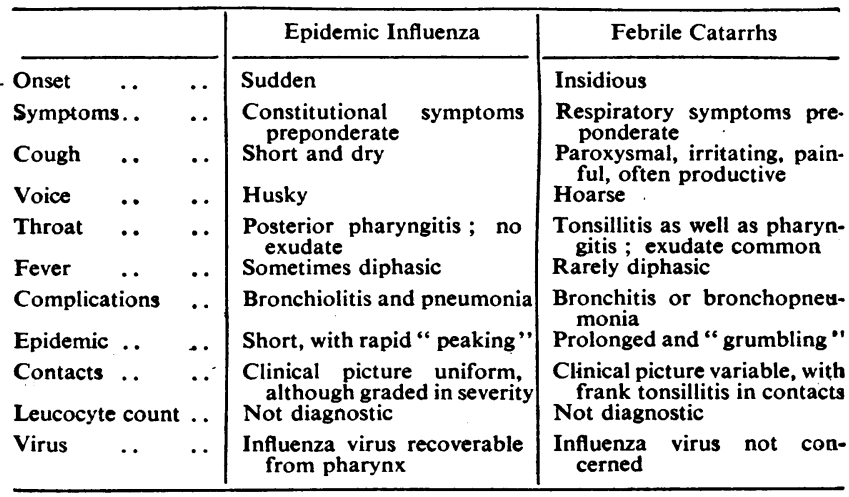

appearance may help, for this is very characteristic in typical influenza, being heavy with drooping eyelids half covering suffused and watering eyes, a dusky malar flush, an obstructed but not running nose, and a furred tongue. The pharyngitis of influenza involves the posterior wall and is dry in contrast with the moist tonsillar and pharyngeal inflammation of febrile catarrh, which often exhibits exudate. Nothing comparable with the bronchiolitis and influenzal pneumonia cases seen recently was encountered in the previous epidemics, although a basal bronchitis or a bronchopneumonia occurs as a complication of febrile catarrh. The blood count was not of help in diagnosis, no definite abnormality being found in the leucocytes in either disease, and the absence of leucopenia, postinfluenzal debility, and depression from the patients in the Services during the recent epidemic was surprising in view of the general belief in these phenomena as criteria of influenza.

In conclusion, I cannot say that it is possible to diagnose epidemic influenza with precision, but I hope I have convinced you of the existence of a clinical entity in that epidemic disease which is associated with the influenza virus.

We are extremely indebted to the Admiralty, the War Office, and the Air Ministry for their permission to work in Service hospitals, and would also like to thank all the officers concerned for their kind co-operation. 